\title{
Novel swirl flow-focusing microfluidic device for the production of monodisperse microbubbles
}

\author{
Irene Arcos-Turmo • Miguel Ángel Herrada • José María López-Herrera • \\ David Fernandez Rivas • Alfonso M. Gañán-Calvo • Elena \\ Castro-Hernández
}

Received: date / Accepted: date

\begin{abstract}
A novel swirl flow-focusing microfluidic axisymmetric device for the generation of monodisperse microbubbles at high production rates to be used as in-line contrast agents for medical applications is presented. The swirl effect is induced upstream of the discharge orifice by a circular array of microblades which form a given angle with the radial direction. The induced vortical component on the focusing liquid stabilizes the gas meniscus by the vorticity amplification due to vortex stretching as the liquid is forced through the discharge orifice. The stabilized meniscus tapers into a steady gas ligament that breaks into monodisperse microbubbles. A reduction up to $57 \%$ in the microbubble diameter is accomplished when compared to conventional axisymmetric flow-focusing microdevices. An exhaustive experimental study is performed for various blade angles and numerous gas to liquid flow rate ratios, validating previous VoF numerical simulations. The microbubbles issued from the stabilized menisci verify prior scaling law of flow-focusing.
\end{abstract}

Keywords microbubble $\cdot$ flow-focusing $\cdot$ swirl

Irene Arcos-Turmo · José María López-Herrera · Alfonso M. Gañán-Calvo · Miguel Ángel Herrada · Elena CastroHernández

Área de Mecánica de Fluidos, Departamento de Ingeniería Aeroespacial y Mecánica de Fluidos, Universidad de Sevilla, Avenida de los Descubrimientos s/n 41092, Sevilla, Spain.

E-mail: elenacastro@us.es

David Fernández Rivas

Mesoscale Chemical Systems and MESA ${ }^{+}$Institute of Nanotechnology, P.O. Box 217, 7500 AE Enschede, The Netherlands.

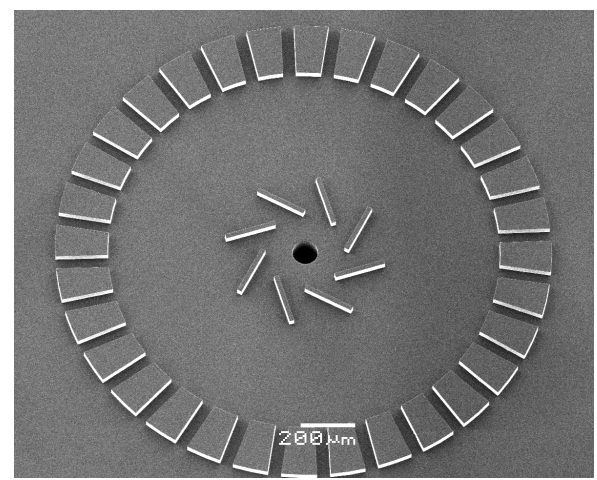

Fig. 1 SEM image of the $60^{\circ}$ blade swirl flow-focusing microfluidic device.

\section{Introduction}

Microbubbles represent not only the obvious counterpart of sprays to diffuse a fluid phase enclosed by surface tension into the environment. Here, the environment is a liquid. Given its enormous inertia combined with high surface tension forces at small scales, the generation, manipulation and dynamics of microbubbles possess unique features not found in any other system. The behavior of microbubbles is often counterintuitive, and generally nonlinear (Marmottant et al, 2005 ). Thus, the physics involved is drastically dominated by very large inertia effects of the environment and the compressibility of the microbubbles. That compressibility combined with their surface properties make them perfect devices for some critical tasks: in biomedical applications, as contrast agents, or as vehicles for drug delivery, or gene therapy by sonoporation (Takahashi, 2005; Ferrara et al, 2007).

As a proxy to qualify the importance of microbubbles as established devices or tools in medicine, one may compare the relative percentages of scientific arti- 
cles published mentioning certain combinations of keywords. For example, one may use public databases to assess that the percentage of papers mentioning "ultrasound", and "microbubbl*" or "micro-bubbl*" over the total has stabilized around $0.024 \%$ over the last ten years. The vast majority of them use the ultrasoundmicrobubble coupled dynamics as the key device to produce benefits in "cardio*" or "cancer*" related applications (Wei et al, 1998; Kennedy et al, 2004). For example, this is comparable to the weight of "amiodarone" , the most widely used antiarrhythmic drug, in cardiology publications $(0.05 \%$ of papers over the last five years), which illustrates the social and economic importance of microbubble-contrast agents in certain medical fields. In this regard, one may verify that the number of papers mentioning "cardio*" and "cancer*" have stabilized at $3.5 \%$ and $6.8 \%$, respectively, over the last hundred years, making them the highest concerns of medicine since long ago. For applications in these fields as contrast agents, to achieve the highest possible efficiency and to minimize gas infusion and adverse effects, reducing the microbubble size and its dispersion as much as possible is of paramount importance: a focused, single frequency excitation is the best way to manipulate swarms of microbubbles in a liquid (generally, blood) stream.

Thus, the search of physical principles and development of technologies to produce the highest possible surface (or minimum microbubble size) per unit input energy, concentrated around a single size value (monodispersity), has fueled an immense collective effort. Almost unfailingly, the solutions proposed for the efficient one-step generation of microbubbles make use of microfluidic designs. In general terms, microfluidics has co-evolved driven by strong expectations in the areas of biomedicine and new materials (Whitesides, 2006). Here, microfluidic devices have demonstrated to be an attractive method to mass-produce narrowly distributed micron size microbubbles in a wide range of liquids (Gañán-Calvo and Gordillo, 2001; Anna et al, 2003; Garstecki et al, 2006). Several microfluidic techniques have been developed in the last decade, being the $\mathrm{T}$ junctions, cross junctions and flow-focusing designs those with the strongest boost.

Despite their differences, two global regimes can be identified: (i) a bubbling regime, where bubbles are formed right at the tip of the injection tube (axisymmetric case) or at the entrance of the outlet channel (planar case), and (ii) a jetting regime characterized by the generation of a jet that breaks up into bubbles (Gañán-Calvo and Gordillo, 2001; Gañán-Calvo, 2004; Gañán-Calvo et al, 2006). These designs can be implemented in axisymmetric geometries (Gañán-Calvo and
Gordillo, 2001), by means of concentric capillary tubes, or in planar configurations using techniques such as soft lithography or micromachining. Bubbles generated by planar flow-focusing devices (Garstecki et al, 2004, 2005) and T junctions (Garstecki et al, 2006; Dollet et al, 2008) have usually diameters of the order of the device geometry as a result of the pinch-off process of either the bubbling or squeezing regimes. It is by forcing both, the liquid and gas streams, through a small aperture or constriction, using flow-focusing (GañánCalvo and Gordillo, 2001) or cross junctions (CastroHernández et al, 2011), that it is possible to achieve bubbles whose size is considerable smaller than the characteristic geometric length. Flow-focusing ensures the production of monodisperse microbubbles at high and controlled production scales. The strong focusing effect created at the constriction induces the formation of a steady tapering gas meniscus, from which bubbles are ejected.

In this work, we propose a robust one-step method to controllably produce small monodisperse microbubbles in an aqueous liquid stream at high production rate, to be employed -among other uses- as contrast agents for medical applications: a novel swirl flow-focusing (SFF) microfluidic device. The essential geometrical difference of our device with respect to all previous implementations is the presence of a circular blade array, concentric to the exit channel, that forces the liquid to swirl around the exit hole (see Figure 1 ). The centrifugal forces created by the swirl originate an intense pressure gradient in the radial direction, stabilizing the gas meniscus and focusing the gas into a short steady gas ligament. Thus, the imposed swirl enables the formation of a tapering meniscus for a wider range of working experimental conditions than in common flow-focusing. This extends the robustness and versatility of co-flow designs to mass produce very small microbubbles to inaccessible parametrical ranges to other known configurations.

\section{Materials and methods}

\subsection{Microfluidic chip design and fabrication}

The SFF microfluidic device creates the swirl effect by forcing the liquid through a circular blade array, concentric to the exit channel and tangent to the liquid flow. Although the microchip design is $3 \mathrm{D}$, the fabrication process is not. Basically, it consists in a regular $2 \mathrm{D}$ engraving into one of the microfluidic chip slabs. Two concentric $50 \mu \mathrm{m}$ filters were placed prior to the blade array to homogenize and filter the liquid flow and to reinforce the rigidity of the chip structure. Based on the 

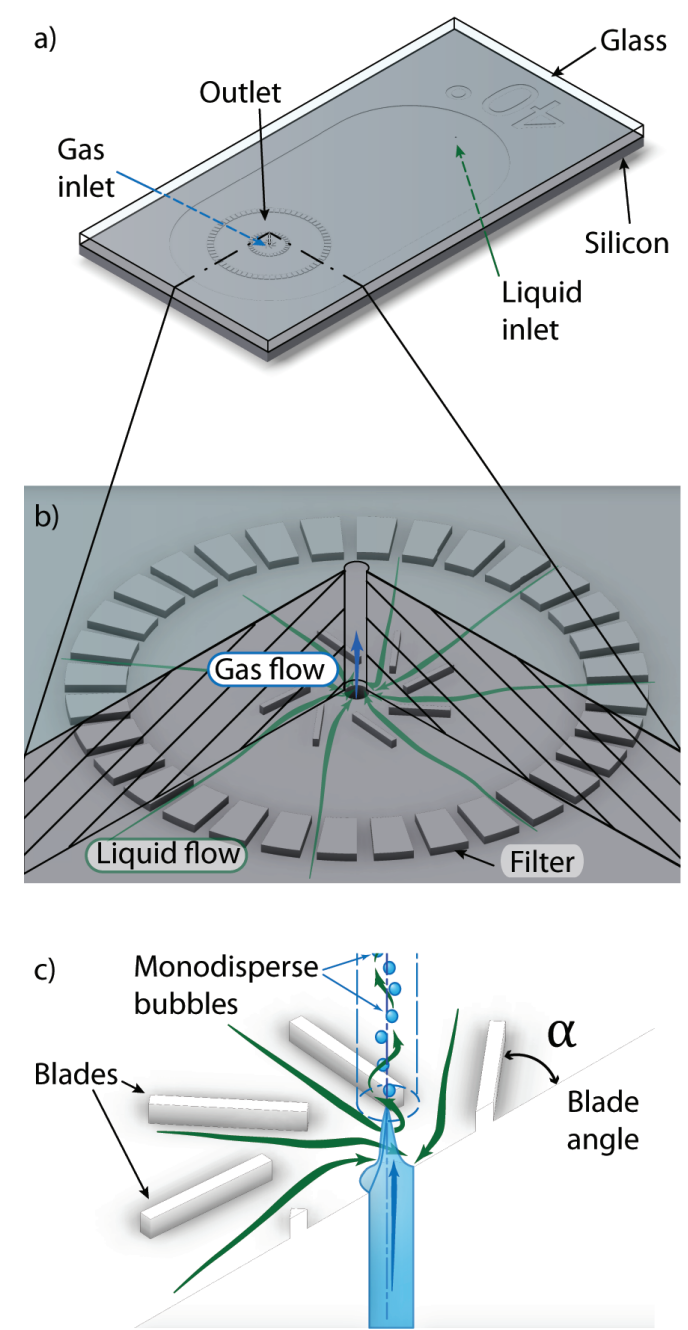

Fig. 2 (a) Sketch of the swirl flow-focusing microfluidic device. (b) Closer view displaying the inner filter and the circular blade array. (c) Sketch of the imposed swirl leading to the formation of a stable gas meniscus that breaks into monodisperse microbubbles.

numerical results obtained using 3D CFD simulations (FLUENT) the following blade parameters were selected: the closest radial position of the blades to the exit channel $R 1=150 \mu \mathrm{m}$, the blade length $L=200 \mu \mathrm{m}$, its width $W=20 \mu \mathrm{m}$, the number of blades $n=8$ and the pith angle $\alpha=0^{\circ}, 40^{\circ}, 60^{\circ}$ and $80^{\circ}$. Rectangular blades were chosen for fabrication simplicity. The blades height was equal to the height of the chamber $H=30 \mu \mathrm{m}$.

The device is made up of a glass wafer $(100 \mathrm{~mm}$ diameter, $1.2 \mathrm{~mm}$ thickness, Borofloat 33; Schott Germany) containing the outlet hole and a silicon wafer (p-type, 5-10 Ohm $\mathrm{cm}$ resistivity, $100 \mathrm{~mm}$ diameter, $525 \mu \mathrm{m}$ thickness, $\{100\}$ crystal orientation; Okmetic Finland) which has both, the gas and the liquid inlet holes, and the microfluidic chamber as shown in Figure 2 .
The $L=1.2 \mathrm{~mm}$ thick Borofloat glass wafer was processed by FEMTOprint SA (Switzerland) using their 3D microstructuring technique to create a hole with a diameter $D=80 \mu \mathrm{m}$, throughout the entire thickness of the glass wafer assuring a perfect alignment between the gas inlet and the emulsion outlet. The use of glass has a double purpose: (i) since $\mathrm{L} / \mathrm{D}=15$, it serves as a microbubble collection channel and (ii) it allows the transversal view of the exit channel.

On the silicon wafer, a $500 \mathrm{~nm}$ thick silicon oxide layer was grown by wet oxidation. Subsequently, the pattern of the microfluidic chamber was transferred via standard photolithography and plasma etching into the silicon oxide layer (Adixen AMS100; Adixen France). The photoresist was removed and a new photolithography step was performed with the mask containing the pattern of the inlet holes. Using deep reactive ion etching (Bosch process, Adixen AMS100; Adixen France) the $80 \mu \mathrm{m}$ inlet holes were etched into the silicon until the silicon oxide on the backside of the wafer was reached. The photoresist was removed and the remaining silicon oxide layer was used as a hard mask to deep reactive ion etch the $H=30 \mu \mathrm{m}$ deep microfluidic chamber. After cleaning, the silicon oxide was removed by etching in 50\% hydrofluoric acid and the wafer was oxidised a second time with the newly formed $1 \mu \mathrm{m}$ thick oxide layer striped afterwards. These last steps were performed in order to remove any residual silicon structures smaller than $1 \mu \mathrm{m}$, which might have remained at the location of the inlet holes due to the combination of the two deep reactive ion etching steps. Prior to bonding, the glass wafer and the silicon wafer were cleaned in a Piranha solution for 15 minutes. The wafer pair was aligned in a mask aligner (EV620 maskaligner; EVG Austria) and the anodic bonding was performed in a vacuum at $400^{\circ} \mathrm{C}$ for 1 hour with 800 Volt applied (EV-501 Anodic Bonder; EVG Austria). As a final step, the bonded wafer stack was diced into chips (Disco DAD 321, Disco Japan) with adhesive foil protecting the in and outlets from contamination.

\subsection{Experimental setup}

The swirl flow-focusing microfluidic device was mounted on a xyz stage for precise translation. A high-speed camera (Shimadzu HPV2) with a resolution of $312 \times 260$ $\mathrm{px}^{2}$ when operated at an acquisition rate of $1 \mathrm{Mfps}$, combined with a flash light source (WalimexPro VC600), was placed perpendicularly to the glass outlet channel.

The continuous phase was Milli-Q water. The surface tension between air and water $\sigma$ was lowered from 72 to $40 \mathrm{mN} / \mathrm{m}$ by adding a $2 \%(\mathrm{w} / \mathrm{v})$ of Tween 80 
(Sigma Aldrich) to the water. The liquid flow rate $Q_{1}$ is controlled by means of a high-precision syringe pump (Model 11 Plus, Harvard Apparatus). The precise control of the gas flow rate $Q_{\mathrm{g}}$ requires imposing a pressure gradient $p_{\mathrm{g}}$ through a pressure regulator (11-818-100, Norgren) and is measured using a digital manometer (Digitron 2030P). To avoid fluctuations in the gas flow rate the air was injected through a fused silica tube with $0.2 \mathrm{~m}$ in length and $75 \mu \mathrm{m}$ of inner diameter. In order to prevent channel clogging by dust particles, an in-line filter (Parker, $0.01 \mu \mathrm{m}$ ) was added to the gas flow line. The swirl flow-focusing chip is connected to the gas and liquid flow lines using peek Nanoports (Assemblies, Nanoport, Upchurch Scientific).

The bubble diameter $d_{\mathrm{b}}$ and bubbling frequency $f_{\mathrm{b}}$ of at least 100 images, are measured via image processing (Image $J$ ). Knowing $d_{\mathrm{b}}$ and $f_{\mathrm{b}}$, the volumetric gas flow rate is determined as $Q_{\mathrm{g}}=\pi d_{\mathrm{b}}^{3} f_{\mathrm{b}} / 6$. For low gas to liquid flow rate ratios, where bubbles tend to have a more elongated shape, the equivalent diameter was likewise calculated.

\section{Results and discussion}

Monodisperse bubbles (polydispersity index PDI 5\%) ranging in size between $6 \mu \mathrm{m}$ and $110 \mu \mathrm{m}$ and at a high production rate $\left(f_{\mathrm{b}} \sim 10^{5} \mathrm{~Hz}\right)$ were generated. Bubbles with diameters below $13 \mu \mathrm{m}$ experience a higher PDI due to the high-speed camera resolution. We accomplished $\sim 1000$ experimental points varying the liquid flow rate from $Q_{1}=0.5 \mathrm{ml} / \mathrm{min}$ to $Q_{1}=1.75$ $\mathrm{ml} / \mathrm{min}$ and selecting gas pressures from $p_{\mathrm{g}}=200 \mathrm{mbar}$ to $p_{\mathrm{g}}=2300 \mathrm{mbar}$, corresponding to flow rate ratios between $Q_{\mathrm{g}} / Q_{\mathrm{l}}=0.01$ and $Q_{\mathrm{g}} / Q_{\mathrm{l}}=1$.

The chosen liquid flow rate range covered both, the bubbling regime (lower values) and the jetting regime (higher values). In presence of high liquid flow rates, specially above $Q_{1}=1.75 \mathrm{ml} / \mathrm{min}$, bubble jet formation becomes increasingly susceptible to perturbations, preventing the meniscus formation due to gas compressibility effects and hydrodynamic feedback. Gas pressure was selected to ensure bubbling and was gradually decreased until no bubbles were ejected.

For the range of liquid flow rates investigated here, $R e=\rho_{\mathrm{l}} v_{\mathrm{b}} D / \mu_{\mathrm{l}} \sim O(700)$, being $\rho_{\mathrm{l}}$ and $\mu_{\mathrm{l}}$ the liquid density and viscosity, respectively, and $v_{\mathrm{b}}$ the velocity of the bubbles at the exit channel. This estimation indicates that the flow at the exit channel is laminar. Figure 3 shows the effect of increasing the liquid flow rate $Q_{1}$ for a $60^{\circ}$ blade SFF microfluidic device and a constant value of the gas pressure $p_{\mathrm{g}}$ confirming the VoF numerical predictions of Herrada and Gañán-Calvo (2009). Increasing the water flow rate results in smaller

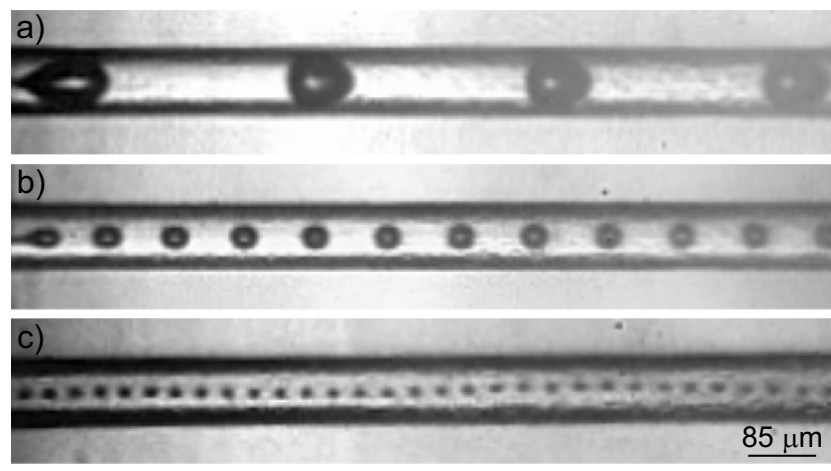

Fig. 3 Series of images showing the effect of increasing the liquid flow rate for a $60^{\circ}$ blade swirl flow-focusing microfluidic device and a constant value of the gas pressure: (a) $Q_{1}=0.5$ $\mathrm{ml} / \mathrm{min}, p_{\mathrm{g}}=851 \mathrm{mbar}, d_{\mathrm{b}}=77.03 \mu \mathrm{m}, f_{\mathrm{b}}=1.01 \times 10^{4}$ $\mathrm{Hz}$; (b) $Q_{\mathrm{l}}=1 \mathrm{ml} / \mathrm{min}, p_{\mathrm{g}}=876 \mathrm{mbar}, d_{\mathrm{b}}=36.57 \mu \mathrm{m}$, $f_{\mathrm{b}}=8.11 \times 10^{4} \mathrm{~Hz}$; (c) $Q_{1}=1.5 \mathrm{ml} / \mathrm{min}, p_{\mathrm{g}}=847 \mathrm{mbar}$, $d_{\mathrm{b}}=13.17 \mu \mathrm{m}, f_{\mathrm{b}}=2.92 \times 10^{5} \mathrm{~Hz}$.

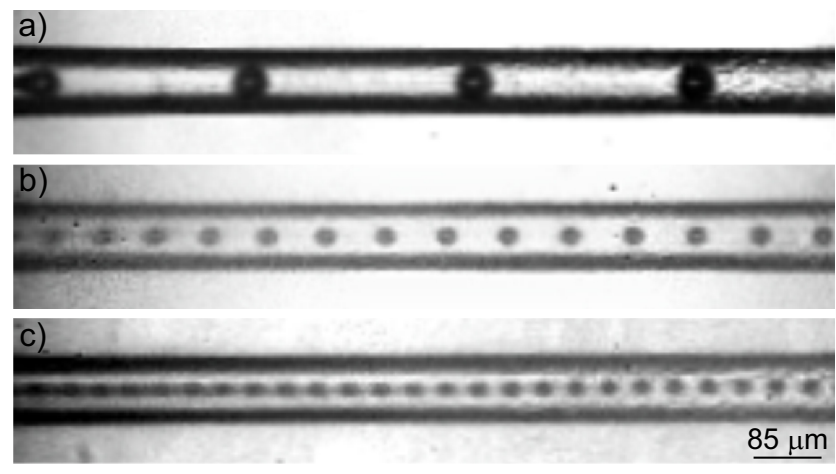

Fig. 4 Series of images showing the effect of increasing the swirl flow-focusing microfluidic device blade angle for a constant gas to liquid flow rate ratio $Q_{\mathrm{g}} / Q_{1}=0.07$ : (a) $0^{\circ}$, $Q_{1}=1 \mathrm{ml} / \mathrm{min}, d_{\mathrm{b}}=45.15 \mu \mathrm{m}, f_{\mathrm{b}}=2.42 \times 10^{4} \mathrm{~Hz} ;(\mathrm{b})$ $40^{\circ}, Q_{1}=1.25 \mathrm{ml} / \mathrm{min}, d_{\mathrm{b}}=28.64 \mu \mathrm{m}, f_{\mathrm{b}}=1.19 \times 10^{5} \mathrm{~Hz}$; (c) $60^{\circ}, Q_{1}=1.5 \mathrm{ml} / \mathrm{min}, d_{\mathrm{b}}=23.72 \mu \mathrm{m}, f_{\mathrm{b}}=2.5 \times 10^{5} \mathrm{~Hz}$.

bubbles and higher breakup frequencies but also narrows the $Q_{1}$ range where monodisperse bubbles can be generated. The same trends were experimentally observed for all the SSF microchip blade angles. Bubbles of $6 \mu \mathrm{m}$ in diameter at a production rate of $2.14 \times 10^{5} \mathrm{~Hz}$ can be obtained when the $60^{\circ}$ blade SFF microdevice is used under the appropriated operating conditions.

Figure 4 displays the effect of increasing the SFF microchip blade angle for a constant value of the gas to liquid flow rate ratio $Q_{\mathrm{g}} / Q_{\mathrm{l}}$. Accordingly to the $\mathrm{VoF}$ numerical simulations presented by Herrada and GañánCalvo (2009), larger values of the SFF microchip blade angle results in smaller bubbles and higher breakup frequencies but also restricts the $Q_{1}$ range where monodisperse bubbles can be generated.

In order to have a reference case for the comparison between different blade angles, a $0^{\circ} \mathrm{SFF}$ device was fabricated. If a conventional FF device is compared with 
- $Q_{l}=0.5 \mathrm{ml} / \mathrm{min}$

- $Q_{l}=1 \mathrm{ml} / \mathrm{min}$

- $Q_{l}=1.25 \mathrm{ml} / \mathrm{min}$

\ $Q_{l}=1.5 \mathrm{ml} / \mathrm{min}$

$\checkmark Q_{l}=1.75 \mathrm{ml} / \mathrm{min}$

a) $\quad S=0$

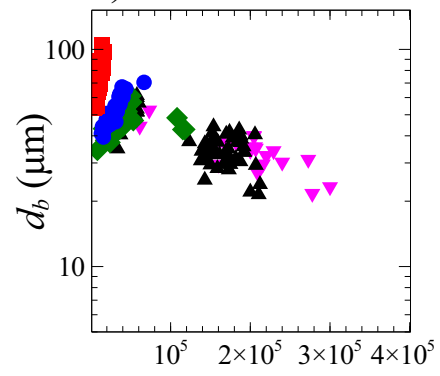

b) $S=0.84$

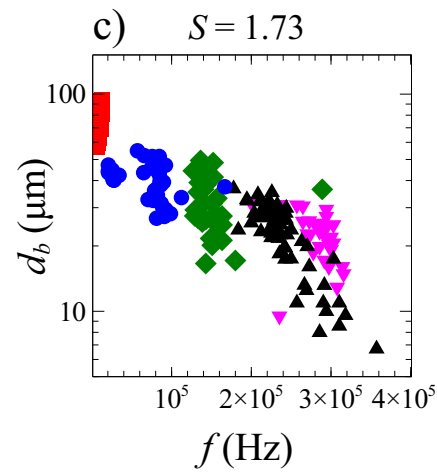

d)

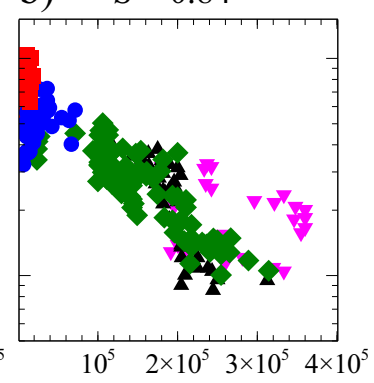

d) $\quad S=5.67$

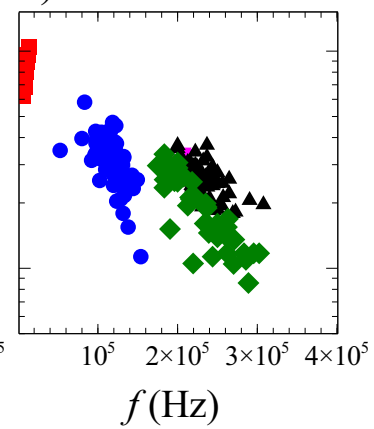

Fig. 5 Bubble diameter versus microbubble production frequency for different blade angles. The indicated swirl factor $S$ is the tangent of the blade angle in this work. The color codes for each liquid flow rate are also indicated.

a SFF, the breakup mechanism and the pressure drop might be completely different and the achieved reduction in bubble size could not be correctly accounted for. A reduction up to $57 \%$ in the microbubble diameter is accomplished when compared to a $0^{\circ} \mathrm{SFF}$.

Figure 5 shows the bubble diameter $d_{\mathrm{b}}(\mu \mathrm{m})$ versus microbubble production frequency $f_{\mathrm{b}}(\mathrm{Hz})$, for different blade angles. The effect of the swirl is clearly shown in this figure: the swirl factor is defined as $S=\tan (\alpha)$. This factor is expected to be approximately concurrent with that defined in Herrada and Gañán-Calvo (2009), since the blade angle $\alpha$ approximately defines the ratio of tangential to radial speeds. According to conservation of mass and angular momentum (excluding the many different boundary layer effects taking place), this ratio is expected to be approximately conserved when the liquid is eventually discharged through the outlet channel. In the absence of swirl (Figure 5(a)) the bubble size is modestly reduced by increasing the frequency of bubbling (i.e. reducing the gas flow rate). In contrast, a drastic decrease in the bubble size can be observed in the presence of swirl (Figure 5(b)) around a critical frequency range, indicating a transition associated to the stabilization of the gas meniscus and the presence of jetting. This is coincident with what was described in Herrada and Gañán-Calvo (2009). One can also observe that the increase of swirl (figures $5(\mathrm{c}) \&(\mathrm{~d})$ ), or the liquid flow rate over certain levels do not necessarily afford much better results in terms of a clear and reproducible decrease of bubble size, owing to the increase flow instabilities and incipient turbulence.

To further represent our results in the framework of prior physical understanding, Figure 6 depicts the microbubble diameter normalized with the exit channel diameter $d_{\mathrm{b}} / D$ as a function of the gas to liquid flow rate ratio $Q_{\mathrm{g}} / Q_{1}$ for different $\mathrm{SFF}$ microchip blade angles $\left(0^{\circ}, 40^{\circ}, 60^{\circ}\right.$ and $\left.80^{\circ}\right)$ and three representative liquid flow rates $\left(Q_{1}=0.5,1\right.$ and $\left.1.5 \mathrm{ml} / \mathrm{min}\right)$.

The figure shows two perfectly differentiable trends related to the two existing regimes. The experimental points corresponding to a bubbling regime, where no stable meniscus is created, are in the upper blue region, above the black solid line. By contrast, the experimental points where the combination of the swirl and flow-focusing effects enables the formation of a stable meniscus are in the lower green region, following the black solid line. In the latter situation, bubbles considerably smaller than the characteristic geometric length are ejected.

The black solid line in Figure 6 represents the scaling law presented by Gañán-Calvo (2004) using conventional axisymmetric flow-focusing devices

$d_{\mathrm{b}} / D=\eta\left(Q_{\mathrm{g}} / Q_{\mathrm{l}}\right)^{0.4}$

where $\eta=1.1$ is a universal constant. In this work, we have found a slight deviation $(\eta=0.9)$ in the coefficient proposed by Gañán-Calvo (2004) due to a vena contracta effect. The high aspect ratio between the chip chamber height $\mathrm{H}$ and the exit channel diameter $\mathrm{D}$, combined with the sharp edge of the entrance of the exit channel leads to a smaller effective exit diameter. The inclusion of the two colored regions constitutes a visual help to easily distinguish between the bubbling and the jetting regimes. The intent is not to give an exact separation (since transitions are never neat) but to be an eye guide for the reader. The straight boundary is chosen accordingly to the power-law fit proposed by GañanCalvo (2004) which is followed by our experiments. The same boundary is used for the three plots in Figure 6 . The figure also manifests that both regimes, bubbling and jetting, can be obtained for a particular gas to liquid flow rate ratio and a given SFF microchip blade angle. Not only is gas to liquid flow rate ratio $\left(Q_{\mathrm{g}} / Q_{\mathrm{l}}\right)$ 

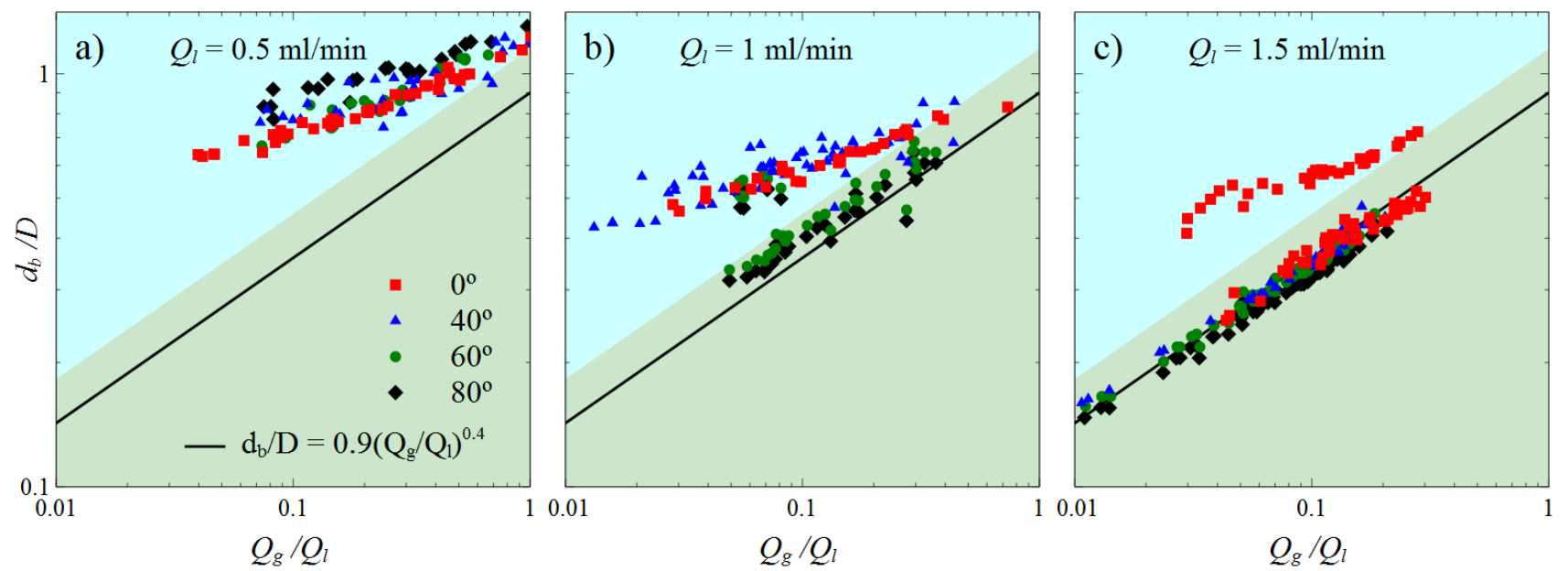

Fig. 6 Dimensionless bubble diameter versus gas to liquid flow rate ratio for different microchip blade angles and increasing values of the liquid flow rate: a) $Q_{1}=0.5 \mathrm{ml} / \mathrm{min}$; b) $Q_{1}=1 \mathrm{ml} / \mathrm{min}$; c) $Q_{1}=1.5 \mathrm{ml} / \mathrm{min}$. The black solid line is the power-law fit proposed by Gañán-Calvo (2004). The upper blue region corresponds to a bubbling regime whereas the lower green region relates to a jetting regime. The colored regions are not an exact boundary but an eye guide for the reader to easily distinguish between the bubbling and the jetting regimes.

relevant to reach a jetting regime, but also their absolute flow rates $\left(Q_{\mathrm{g}}\right.$ and $\left.Q_{1}\right)$. For instance, in Figure 6 (c) for a gas to liquid flow rate ratio $Q_{\mathrm{g}} / Q_{1}=0.1$ we have two completely different experimental points: (i) a microbubble produced in the bubbling regime with a dimensionless bubble diameter $d_{\mathrm{b}} / D=0.57$, a gas flow rate $Q_{\mathrm{g}}=0.15 \mathrm{ml} / \mathrm{min}$, a liquid flow rate $Q_{1}=1.5$ $\mathrm{ml} / \mathrm{min}$ and a production rate $f_{\mathrm{b}}=4.16 \times 10^{4} \mathrm{~Hz}$ and (ii) a microbubble produced in the jetting regime with a dimensionless bubble diameter $d_{\mathrm{b}} / D=0.37$, a gas flow rate $Q_{\mathrm{g}}=0.14 \mathrm{ml} / \mathrm{min}$, a liquid flow rate $Q_{1}=1.5$ $\mathrm{ml} / \mathrm{min}$ and a production rate $f_{\mathrm{b}}=1.42 \times 10^{5} \mathrm{~Hz}$. The transition between both regimes is a delicate boundary that can be crossed with a change in gas pressure of just a few milibars. This sensitivity to small changes is especially present for a blade angle equal to $0^{\circ}$. As soon as the blade angle increases, the possibility of two different regimes for similar operating conditions disappears, which strongly reinforces the convenience of the swirl flow-focusing configuration.

The transition from bubbling to jetting regime can be accomplished (for a fixed geometry and fluids) by increasing the liquid flow rate, as it was previously seen in Figure 3. Our experimental study demonstrates that this transition can also be conducted imposing a swirl on the liquid. Both effects are comprised in Figure 6: the increase of liquid flow rate and microchip blade angle. Furthermore, the stronger the swirl is, i.e. higher the blades angle, the lower liquid flow rates are needed to work on jetting conditions. Thus, the shift to jetting is reached more easily thanks to the imposed swirl. The transition occurs around $Q_{1}=1.5 \mathrm{ml} / \mathrm{min}$ using $0^{\circ}$



b)

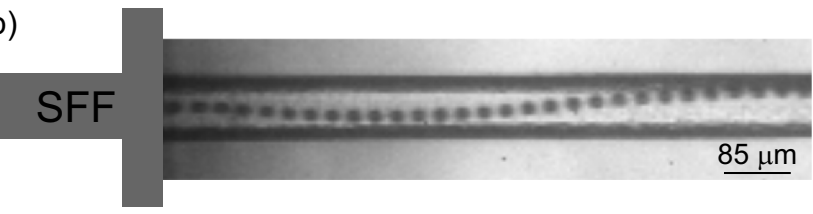

Fig. 7 (a) Numerical simulation illustrating an undulating bubble train. (b) Image showing the experimental undulating bubble train for a $40^{\circ}$ blade angle SFF microdevice, $Q_{1}=1.75$ $\mathrm{ml} / \mathrm{min}, p_{\mathrm{g}}=1341 \mathrm{mbar}, d_{\mathrm{b}}=18.45 \mu \mathrm{m}, f_{\mathrm{b}}=3.6 \times 10^{5} \mathrm{~Hz}$.

blade angle chips, but decreases to $1.25 \mathrm{ml} / \mathrm{min}$ for $40^{\circ}$ blade angle microchips and to $1 \mathrm{ml} / \mathrm{min}$ for $60^{\circ}$ and $80^{\circ}$ microdevices. We observed a plateau on the swirl general performance above $60^{\circ}$ blade angle, related to the previously observed drastic decrease of the effect of swirl over an optimal strength (see Herrada and GañánCalvo (2009)). Part of that decrease could be attributed to the enhancement of perturbations.

Figure 7 shows the bubble train undulation predicted by the VoF numerical simulations and its experimental observation. The simulation was previously obtained by Herrada and Gañán-Calvo (2009) for pure water (without surfactant) and imposing numerically a swirl on a conventional axisymmetric Flow-Focusing 
device. This undulation is due to experimental perturbations, such as dust particles, that may disrupt the axial symmetry of the jet. It also confirms that there is no bubble size change despite the trajectory deviation, which was forced in the simulations and naturally occurred in some experimental cases. The helicoidal trace followed by the bubbles is the result of the imposed liquid swirl, migrating the bubbles towards the regions of lower pressure at the axis of the liquid vortex.

Lastly, experiments with ultra-pure MiliQ water (without surfactant) were also conducted. The results showed an overall similar behavior, although the increase of surface tension favored the presence of hydrodynamic feedback. Consequently, the accessible flow rate ratios range was drastically reduced.

\section{Conclusions}

A novel swirl flow-focusing microfluidic device for the production of monodisperse microbubbles at a high production rate is presented. The swirl effect is achieved thanks to the rotation induced by a circular array of microblades turned a certain angle. The addition of a swirl component into the focusing liquid stabilizes the gas meniscus from which a steady gas ligament issues breaking into monodisperse microbubbles. Furthermore, the swirl is shown to expand the bounds of the jetting mode inhibiting the dripping mode. As consequence of the extension of the jetting regime, a reduction up to $57 \%$ in the microbubble diameter is accomplished when compared to conventional axisymmetric flow-focusing microdevices.

Inspired by the numerical results of Herrada and Gañán-Calvo (2009) and Herrada et al (2011), we perform 3D CFD simulations (FLUENT) to determine the most promising geometric dimensions for the swirl flowfocusing microdevices. Based on the simulations, silicon microchips with blade angles $0^{\circ}, 40^{\circ}, 60^{\circ}$ and $80^{\circ}$ were fabricated. Working under the appropriate experimental conditions, monodisperse bubbles (PDI $\sim 5 \%$ ) ranging in size between $6 \mu \mathrm{m}$ and $110 \mu \mathrm{m}$ and at high production rate $\left(f_{\mathrm{b}} \sim 10^{5} \mathrm{~Hz}\right)$ can be generated. An exhaustive experimental study $(\sim 1000$ experimental points) is performed validating previous VoF numerical simulations and complying with the flow-focusing scaling law proposed by Gañán-Calvo (2004). The $60^{\circ}$ swirl flow-focusing microfluidic device shows the best performance, among our tested chips, with a trade off between swirl effect and robustness against perturbations.

Acknowledgements The authors would like to acknowledge financial support from Spanish Government Ministry MEIC and Regional Government under the Contract DPI2013-46485 and P11-TEP-7465, respectively. They would also like to acknowledge the technical assistance of S. Schlautmann in the fabrication of the microfluidic devices and Manuel González and Jorge López for their technical assistance during the setup preparation.

\section{References}

Anna SL, Bontoux N, Stone HA (2003) Formation of dispersions using flow focusing in microchannels. Applied Physics Letters 82(3):364-366

Castro-Hernández E, van Hoeve W, Lohse D, Gordillo JM (2011) Microbubble generation in a co-flow device operated in a new regime. Lab on a Chip 11(12):2023-2029

Dollet B, Van Hoeve W, Raven JP, Marmottant P, Versluis M (2008) Role of the channel geometry on the bubble pinch-off in flow-focusing devices. Physical Review Letters 100(3):034504

Ferrara K, Pollard R, Borden M (2007) Ultrasound microbubble contrast agents: Fundamentals and application to gene and drug delivery. Annual Review of Biomedical Engineering 9:415-447

Gañán-Calvo AM (2004) Perfectly monodisperse microbubbling by capillary flow focusing: An alternate physical description and universal scaling. Physical Review E 69(2):027301

Gañán-Calvo AM, Gordillo JM (2001) Perfectly monodisperse microbubbling by capillary flow focusing. Physical Review Letters 87(27):274501

Gañán-Calvo AM, Herrada MA, Garstecki P (2006) Bubbling in unbounded coflowing liquids. Physical Review Letters 96(12):124504

Garstecki P, Gitlin I, Diluzio W, Kumacheva E, Stone HA, Whitesides GM (2004) Formation of monodisperse bubbles in a microfluidic flow-focusing device. Applied Physics Letters 85:2649-2651

Garstecki P, Gañán-Calvo A, Whitesides G (2005) Formation of bubbles and droplets in microfluidic systems. Technical Sciences 53(4)

Garstecki P, Fuerstman MJ, Stone HA, Whitesides GM (2006) Formation of droplets and bubbles in a microfluidic t-junctionñscaling and mechanism of breakup. Lab on a Chip 6(3):437-446

Herrada MA, Gañán-Calvo AM (2009) Swirl flow focusing: A novel procedure for the massive production of monodisperse microbubbles. Physics of Fluids 21(4):042003

Herrada MA, Gañán-Calvo AM, López-Herrera JM (2011) Generation of small mono-disperse bubbles in axisymmetric t-junction: the role of swirl. Physics of Fluids 23(7):072004 
Kennedy J, Wu F, Ter Haar G, Gleeson F, Phillips R, Middleton M, Cranston D (2004) High-intensity focused ultrasound for the treatment of liver tumours. Ultrasonics 42(1-9):931-935

Marmottant P, Van Der Meer S, Emmer M, Versluis M, De Jong N, Hilgenfeldt S, Lohse D (2005) A model for large amplitude oscillations of coated bubbles accounting for buckling and rupture. Journal of the Acoustical Society of America 118(6):3499-3505

Takahashi M (2005) Potential of microbubbles in aqueous solutions: Electrical properties of the gas - water interface. Journal of Physical Chemistry B 109(46):21858-21864

Wei K, Jayaweera A, Firoozan S, Linka A, Skyba D, Kaul S (1998) Quantification of myocardial blood flow with ultrasound-induced destruction of microbubbles administered as a constant venous infusion. Circulation 97(5):473-483

Whitesides G (2006) The origins and the future of microfluidics. Nature 442(7101):368-373 